\title{
Severe hypertension in pregnancy: Using dynamic checklists to save lives
}

\author{
J Moodley, ${ }^{1}$ MB ChB, FRCOG, FCOG, MD; N C Ngene, ${ }^{2}$ Dip Obst, Dip HIV Man, MMed (Fam Med), FCOG, MMed (O\&G) \\ ${ }^{1}$ Women's Health and HIV Research Group, Department of Obstetrics and Gynaecology, School of Clinical Medicine, College of Health Sciences, \\ Nelson R Mandela School of Medicine, University of KwaZulu-Natal, Durban, South Africa \\ ${ }^{2}$ Department of Obstetrics and Gynaecology, School of Clinical Medicine, College of Health Sciences, Nelson R Mandela School of Medicine, \\ University of KwaZulu-Natal, Durban, South Africa
}

Corresponding author: J Moodley (jmog@ukzn.ac.za)

\begin{abstract}
Severe hypertension is a major cause of morbidity and mortality. The South African Saving Mothers report (2011 - 2013) indicates that cerebral injury due to severe hypertension is resulting in avoidable maternal deaths. This demands that management of severe hypertension in pregnancy needs to be improved. A rapid-acting antihypertensive is recommended for the initial management of severe hypertension during pregnancy. A single dose of a rapid-acting agent may be ineffective, in which case incremental doses of the same medication or another antihypertensive may be required for adequate blood pressure control. To ensure that appropriate antihypertensives at the correct doses are administered, the use of a guideline in a dynamic checklist format is advocated and discussed in this article. It is envisaged that the use of dynamic checklists will be valuable to all healthcare professionals providing care during pregnancy and the puerperium.
\end{abstract}

S Afr Med J 2016;106(8):767-770. DOI:10.7196/SAMJ.2016.v106i8.10908

Hypertensive disorders of pregnancy (gestational hypertension, pre-eclampsia, eclampsia and chronic hypertension) are a major cause of maternal mortality in South Africa (SA) and worldwide. The most recent Saving Mothers report $(2011-2013)^{[1]}$ indicates that $14.8 \%$ of all maternal deaths were due to hypertensive disorders of pregnancy (HDP). This publication, which is the triennial report of the National Committee for Confidential Enquiries into Maternal Deaths (NCCEMD) in SA, also indicates that the final cause of death in $>50 \%$ of hypertensive maternal mortality was probably associated with cerebral injury, particularly in cases of severe pre-eclampsia and eclampsia. In addition, the majority of deaths occurred in young women in their first pregnancies and a large proportion $(>60 \%)$ were avoidable. ${ }^{[1]}$

Although severe HDP may cause other target organ damage such as renal impairment and pulmonary oedema, the authors have chosen to elaborate on cerebral injury, given the Saving Mothers report on the association between untreated severe hypertension and cerebral haemorrhage. ${ }^{[1]}$ Moreover, in the authors' clinical experience and according to findings in recent reports, severe hypertension often precedes other cerebral manifestations such as blindness in pregnancy, stroke, altered mental state and the posterior reversible encephalopathy syndrome (PRES)..$^{[2-6]}$

\section{PRES and severe hypertension in preginancy}

A known maternal cerebral complication of severe pre-eclampsia is PRES. ${ }^{[2]}$ This syndrome is a clinico-neuroimaging entity and is hypothesised to be related to impaired cerebral blood flow autoregulation that leads to either over- or under-perfusion of the brain. On magnetic resonance imaging and computed tomography scans of the brain, oedema is seen mainly in the subcortical white matter and occasionally in the cortex of the occipital and parietal lobes. ${ }^{[3]}$ Recently, Van Veen et al. ${ }^{[4]}$ investigated cerebral blood flow autoregulation by measuring cerebral artery blood flow velocity using transcranial Doppler ultrasound and found impaired autoregulation in women with pre-eclampsia when compared with those who had gestational hypertension or normal pregnancies. There is therefore increasing evidence that impaired cerebral blood flow autoregulation plays a central pathological role in cerebral manifestations associated with pre-eclampsia/eclampsia syndrome.

Symptoms of PRES include persistent headache, nausea, vomiting, visual disturbances, confusion and seizures. These clinical signs are well known by obstetricians to herald seizures and other features of cerebral dysfunction. Eclampsia is reported to be one of the most important causes of PRES, and although most patients have severe hypertension, some have mildly elevated or even normal blood pressure (BP)..$^{[2,4]}$

It has been reported that PRES is reversible and that initiating early treatment of severe hypertension leads to cure, while delayed recognition of symptoms and cognisance of the need to lower high BP judiciously but immediately leads to cerebral injury. ${ }^{[2,3]}$ The Saving Mothers report $(2011-2013)^{[1]}$ indicates that one of the avoidable health professional factors leading to maternal mortality is failure to institute appropriate and prompt management of severe systolic and diastolic hypertension in the ante-, intra- and postpartum periods.

\section{Immediate treatment of severe hypertension in pregnancy}

Standardised clinical protocols for the management of severe preeclampsia and eclampsia have been shown to reduce complications associated with this hypertensive disorder. ${ }^{[7-9]}$ In pregnancy, acute-onset severe hypertension that is accurately measured using standard techniques and persistent for $\geq 15$ minutes is regarded as a hypertensive emergency. ${ }^{[7-9]}$ If not adequately treated, severe hypertension can cause cerebral injury. A systolic BP of $\geq 160 \mathrm{mmHg}$ is included in the definition of severe hypertension in pregnancy. Severe diastolic hypertension of $\geq 110 \mathrm{mmHg}$ is also regarded as a hypertensive emergency. ${ }^{[7-10]}$ Pregnant women with acute-onset, severe systolic and/or diastolic BPs in the ante-, intra-, or immediate 
postpartum periods therefore warrant antihypertensive treatment. The goal should be to lower high BP levels to a range of 140 - 150/90 $100 \mathrm{mmHg}$ in order to prevent repeated prolonged exposure of the patient to severe systolic hypertension with subsequent loss of cerebral vasculature autoregulation. ${ }^{[10]}$

\section{Some case scenarios and the ' 5 Rs' in severe hypertension in pregnancy}

If health professionals are aware that severe hypertension in pregnancy may result in cerebral injury, what should they do when they are faced with such clinical situations in an SA setting?

Health professionals must be aware of the $\mathbf{5}$ Rs:

1. Recognition that acute-onset severe hypertension ( $\geq 160 \mathrm{mmHg}$ systolic or $\geq 110 \mathrm{mmHg}$ diastolic BP) in pregnant women is a MAJOR ALERT

2. Responding by notifying a specialist or seeking advice from the most appropriate health professional

3. Responsibility: taking responsibility to initiate early antihypertensive therapy and provide close monitoring until the patient is stabilised

4. Reviewing the clinical situation once high $\mathrm{BP}$ is stabilised

5. Realising that effective high BP control is merely a surrogate marker of maternal and neonatal outcome; components such as fetal assessment, prevention of seizures (eclampsia), expeditious delivery and post-delivery care must therefore be considered. ${ }^{[1-10]}$

\section{Case scenarios}

Health professionals may be faced with women with acute-onset severe hypertension in varying clinical scenarios. These include:

- In the antenatal period. When hypertension occurs in this setting, the patient should be admitted to hospital immediately and the high BP should be stabilised before delivery, even in urgent situations. ${ }^{[1]}$ Lowering of the high BP should occur in a high-care area, or a dedicated bed selected for this purpose.

- If transfer from a district health facility to a regional/tertiary centre is required, the high BP should be stabilised and other measures such as the administration of magnesium (in cases of severe preeclampsia and/or eclampsia) started before transfer. Monitoring must continue while awaiting an ambulance for the transfer.

- When the diagnosis of acute-onset severe pre-eclampsia is made in an office setting, available emergency treatment should be initiated and the patient expeditiously sent to a regional/tertiary hospital for treatment and further management.

- If any hospitalised patient with pre-eclampsia has either a systolic BP of $160 \mathrm{mmHg}$ or a diastolic BP of $110 \mathrm{mmHg}$, the automatic response should be initiating a rapid-acting antihypertensive agent such as nifedipine $10 \mathrm{mg}$ orally, labetalol $20 \mathrm{mg}$ intravenously (IV) or hydralazine 5 - $10 \mathrm{mg}$ IV. ${ }^{[9,10]}$

- Induction of anaesthesia and intubation. In a setting in which the patient may require endotracheal intubation in a labour ward (high-care bed), an operating theatre or an intensive care unit, it should be recognised that endotracheal intubation increases the BP and should therefore not be undertaken without prior measures to prevent or minimise the hypertensive response to intubation.

- Acute-onset severe hypertension in the postpartum period. Onset may occur for the first time after delivery, or it may be superimposed on women with chronic hypertension, gestational hypertension and those with mild to moderate pre-eclampsia. In women with such diagnoses, long-acting antihypertensive therapy must therefore be maintained after delivery and not stopped abruptly. In the clinical setting of postpartum acuteonset severe hypertension, the same treatment, viz. rapid-acting antihypertensive agents, must be used to stabilise systolic and/or diastolic hypertension.

In general terms, healthcare professionals must provide close maternofetal monitoring during the stabilisation of acute-onset severe hypertension. Judicious fluid administration is recommended, especially in the oedematous pre-eclamptic patient with oliguria and laboratory signs of renal dysfunction. Fluid balance and signs of early pulmonary oedema must therefore be given careful attention. After initial BP stabilisation, close monitoring should be carried out and a maintenance dose of antihypertensive(s) given.

Over the past decade, the use of clinical protocols and dynamic checklists has become a standard approach to ensure patient safety and improve the care provided to mothers and their newborns. ${ }^{[11]}$ The clinical management of severe acute-onset pregnancy hypertension lends itself to dynamic checklists.

\section{First-line antilhypertensive therapy for} acuite-onset severe hypertension

IV labetalol, IV dihydralazine and oral nifedipine are the commonly used antihypertensive agents. The contraindications to and compelling indications for the available rapid-acting agents must be considered when choosing a particular drug. Magnesium sulphate is not recommended as an antihypertensive agent, but remains the drug of choice for seizure prophylaxis in severe pre-eclampsia and for controlling seizures in eclampsia. ${ }^{[12]}$ Nifedipine may be the more appropriate antihypertensive agent in a setting of primary healthcare clinics and midwifery obstetric units because it can be given orally. ${ }^{[13,14]}$ Intravenous agents (labetalol or dihydralazine if available) are preferable if the patient is restless or semiconscious or in a hospital setting.

Fig. 1 outlines the steps for the use of rapid-acting antihypertensive agents for acute-onset severe hypertension during pregnancy and the postpartum period.

It should be noted that dihydralazine is not available in most provinces of SA. Oral nifedipine and/or labetalol should therefore be a 'stock item' for all hospitals providing healthcare for pregnant women. Both of these drugs are available in public sector hospitals in SA. The common contraindications of rapid-acting antihypertensive agents are shown in Table 1. All the agents listed may cause severe hypotension, but these events are rare. It is suggested that where possible fetal heart rate monitoring should be done at the same time as the lowering of very high BP.

\section{Key points}

- There is consensus that sustained severe hypertension in pregnancy should be lowered immediately in a controlled manner to reduce the risk of cerebral complications.

- A systolic BP of $160 \mathrm{mmHg}$ is suggested as the most appropriate threshold for defining severe maternal hypertension.

- If threshold systolic BP levels are sustained or occur with a combination of symptoms suggesting maternal cerebral complications, immediate use of rapid-acting antihypertensive agents is essential.

- The goal of rapid-acting antihypertensive agents should be to achieve a BP of $140-150 / 90-100 \mathrm{mmHg}$.

- Use of dynamic checklists is essential for the management of acuteonset severe pregnancy hypertension.

\section{Conclusion}

Severe hypertension in pregnancy is an emergency (major alert) that requires immediate attention. An inappropriately managed severe 


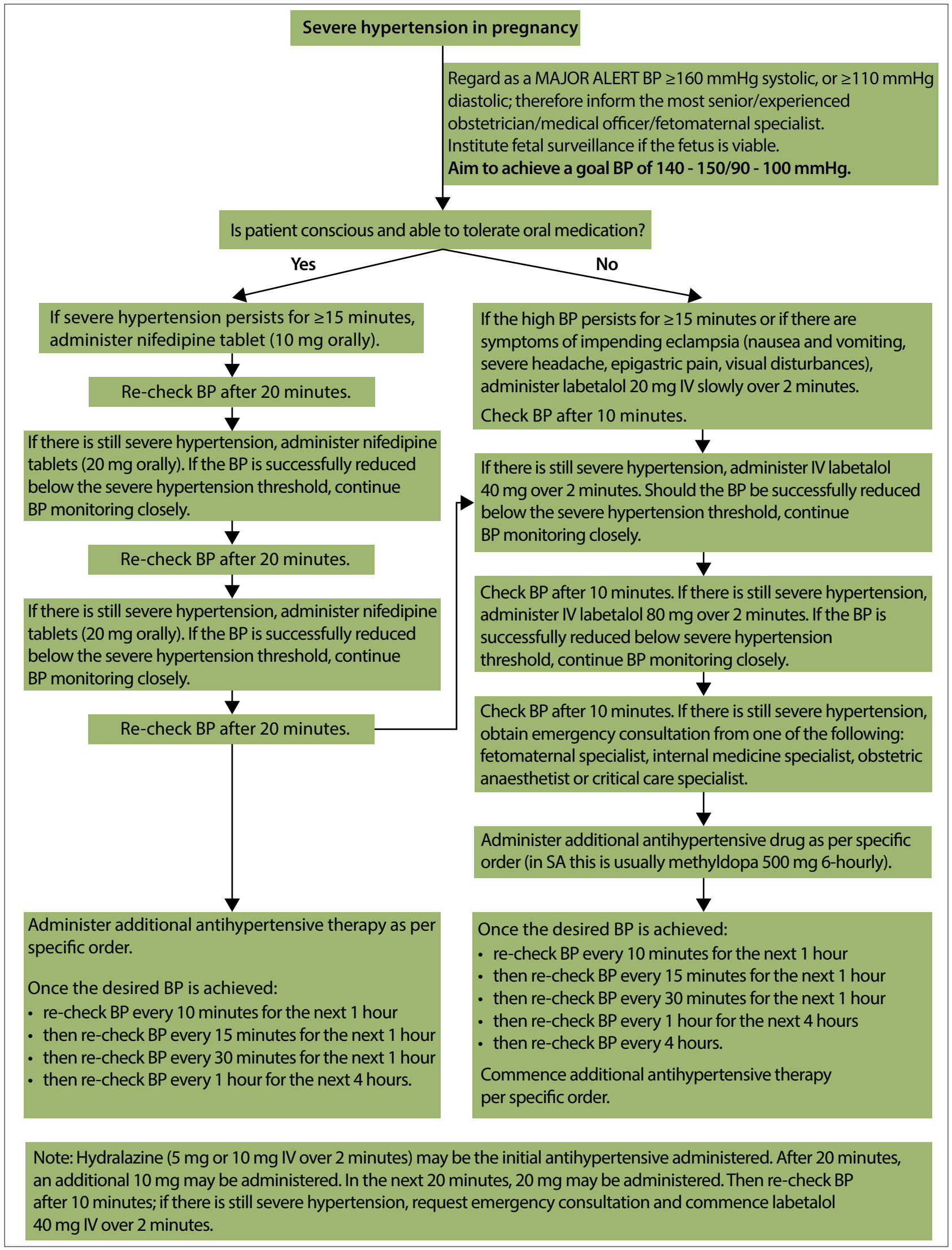

Fig. 1. Algorithm for the initial control of BP in severe hypertension in pregnancy.

hypertensive condition may result in cerebral haemorrhage. Timely action to reduce sustained systolic hypertension judiciously by means of a rapid-acting antihypertensive drug will reduce maternal and neonatal mortality and morbidity. 
Table 1. Common contraindications of the rapid-acting antihypertensive agents

\begin{tabular}{lll}
\hline Cautions & Contraindications \\
\hline Nifedipine & $\begin{array}{l}\text { Hypertrophic obstructive cardiomyopathy, aortic stenosis. The } \\
\text { capsules cause a reflex increase in sympathetic tone which } \\
\text { should be avoided in women with increased myocardial oxygen } \\
\text { demands, e.g. fixed valvular obstruction. }\end{array}$ & Unstable angina, congestive heart failure, myocardial infarction \\
Labetalol & $\begin{array}{l}\text { Controlled heart failure, hepatic disorder. Best avoided in } \\
\text { women with asthma. Parenteral labetalol may cause neonatal } \\
\text { bradycardia, but is not a major problem in clinical practice. }\end{array}$ & $\begin{array}{l}\text { Heart failure with symptoms, 2nd and 3rd degree } \\
\text { atrioventricular block, severe bronchospasm, asthma, sinus } \\
\text { bradycardia }\end{array}$ \\
Hydralazine & $\begin{array}{l}\text { Renal or hepatic impairment, porphyria, angina. It causes a reflex } \\
\text { increase in sympathetic tone and should therefore be avoided for } \\
\text { women in whom increased myocardial oxygen demands could be } \\
\text { dangerous, e.g. coronary artery disease, mitral stenosis. }\end{array}$ & Sypertrophic obstructive cardiomyopathy \\
&
\end{tabular}

1. National Committee on the Confidential Enquiries into Maternal Deaths. Saving Mothers 20112013: Sixth Report on the Confidential Enquiries into Maternal Deaths in South Africa. Short report. Pretoria: NDoH, 2015. http://www.kznhealth.gov.za/mcwh/Maternal/Saving-Mothers-2011-2013Pretoria: NDoH, 2015. http://www.kznh
short-report.pdf (accessed 28 August 2015).

2. Mayama M, Uno K, Tano S, et al. Incidence of posterior reversible encephalopathy in eclamptic and patients with preeclampsia with neurologic symptoms. Am J Obstet Gynecol 2016; Feb 20. pii: S0002patients with preeclampsia with neurologic symptoms. Am J Obstet Gyn

9378(16)00339-2. DOI:10.1016/j.ajog.2016.02.039 [Epub ahead of print]
3. Naidu K, Moodley J, Corr P, Hoffmann M. Single photon emission and cerebral

3. Naidu K, Moodley J, Corr P, Hoffmann M. Single photon emission and cerebral computerised tomographic scan and TCD in eclampsia. BJOG 1997;104(10):1165-1172. DOI:10.1111/.1471-0528.1997.tb10941.x

4. Van Veen TR, Panerai RB, Haeri S, et al. Cerebral autoregulation in different hypertensive disorders of pregnancy. Am J Obstet Gynecol 2015;513:e3. DOI:10.1016/j.ajog.2014.11.003

5. Crovetto F, Somalglaiana E, Peguero A, Figueras F. Stroke during pregnancy and pre-eclampsia. Curr Opin Obstet Gynecol 2013;25(6):425-432. DOI:10.1097/GCO.0000000000000024

6. Bushnell C, Chireau M. Pre-eclampsia and stroke. Stroke Res Treat 2011 (2011), Article ID 858134 DOI:10.4061/2011/858134

7. Von Dadelszen P, Sawchuck D, McMaster R, et al. The active implementation of pregnancy hypertension gudelines in British Columbia Obstet Gynecol 2010:116(3):659-666. DOI:10.1097/ AOG.0b013e3181eb669d.

8. American College of Obstetricians and Gynecologists. Emergent therapy for acute-onset, severe hypertension during pregnancy and the postpartum period. Committee opinion No. 623. Obstet Gynecol 2015;125(2):521-525. DOI:10.1097/01.AOG.0000460762.59152.d7
9. Magee LA, Abalos E, von Dadelszen P, et al., for the CHIPS Study Group. How to manage hypertension in pregnancy effectively. Br J Clin Pharmacol 2011;72(3):394-401. DOI:10.1111/j.13652125.2011.04002.x

10. Clark SL, Christmas JL, Frye DR, Meyers JA, Perlin JR. Maternal mortality in the United States: Predictability and the impact of protocols on fatal post caesarean pulmonary embolism and hypertension-related intracranial haemorrhage. Am J Obstet Gynecol 2014;211(1):32.e1-32e9. hypertension-related intracranis

11. Fausett MB, Propst A, von Doren K, Clark BT. How to develop an effective obstetric checklist. Am J Obstet Gynecol 2011;205(3):165-170. DOI:10.1016/.jajog.2011.06.003.

12. Altman D, Carroli G, Duley L, et al., for Magpie Trial Collaboration Group. Do women with pre-eclampsia and their babies benefit from magnesium sulphate? The Magpie Trial: A randomised placebo-controlled trial. Lancet 2002;359(9321):1877-1890. DOI:10.1016/S01406736(02)908778-0.

13. Shekhar S, Gupta S, Kirubakaran R, Pareek P. Oral nifedipine versus intravenous labetalol for severe hypertension during pregnancy: A systematic review and meta-analysis. BJOG 2016;123(1):40-47. DOI: $10.1111 / 1471-0528.13463$

14. Duley L, Meher S, Jones L. Drugs for treatment of very high blood pressure during pregnancy. Cochrane Database Syst Rev 2013, Issue 7. Art. No.: CD001449. DOI:10.1002/14651858.cd001449. pub3

Accepted 13 April 2016. 